\section{ALGUNOS APUNTES SOBRE LAS RAZONES DE LA REFORMA DEL PROCEDIMIENTO PENAL EN AMÉRICA LATINA}

\section{Pierre Gilles Bélanger*}

Fecha de recepción: 19 de Octubre de 2010

Fecha de aceptación: 30 de Noviembre de 2010 Artículo de Reflexión.

\section{Resumen}

Actualmente en Latinoamérica, la mayor parte de los países han modificado sus códigos penales con el objeto de tener una mejor salud procesal. En esta transición, hace falta ayuda internacional real, apoyo, colaboración e intercambio de ideas, programas, experiencias, grupos de trabajo, capacitación a nivel bilateral y multilateral dirigida por expertos para los actores en el sistema penal y para la sociedad. Todos los países de Latinoamérica, han tenido éxitos y todos tienen fallas en las reformas procesales desarrolladas en materia penal. Parece

El autor es miembro de la Barra de Abogados de Quebec. De 2000 a 2007 ha sido para Canadá el primer coordinador de un nuevo grupo de trabajo formado por expertos en las Américas sobre la cooperación jurídica en materia penal para la Organización de los Estados Americanos (OEA). En 2008, el Ministerio de Justicia de Canadá lo destacó al Centro de Estudios de la Justicia de las Américas (CEJA), situado en Santiago de Chile. Este Centro se especializa en reforma penal en las Américas. Actualmente el autor se encuentra en el Tecnológico de Monterrey, México, Campus Cuernavaca, donde está impartiendo dos cursos en la carrera de Relaciones Internacionales sobre América Latina, el Caribe y América del Norte. 2010 Certification - Tecnológico de Monterrey, Monterrey, México - Reforma Penal; 1995 M.A. Master in Political Thought - Philosophy of Law, University of Ottawa, Thesis: A critique of the Canadian judicial system; 1989 Québec Bar School; 1989 LL.L. - University of Ottawa; 1985 B.S.Sc. Honours Political Science - University of Ottawa; 1983 B.A. in Psychology - University of Ottawa. E1 autor agradece la colaboración de Raúl R. Llorente Abogado en México y L.L.B. en Canadá. pbelange@ justice.gc.ca entonces oportuno, empezar el dialogo y la colaboración para contribuir a una mejor comprensión y edificación de los sistemas penales y su evolución. Para ello se requiere compartir las experiencias que han funcionado en otros países, adaptándolas al marco jurídico de cada contexto en particular. Por un lado, esto ayuda a mejorar la comprensión operacional del procedimiento penal y por otro lado, el respeto de las garantías jurídicas establecidas en las Cartas Magnas de cada país y por ende estabilidad institucional, seguridad y desarrollo económico para la sociedad.

\section{Palabras clave}

Reforma procesal, razones y limitaciones, códigos criminales, cooperación internacional, derechos fundamentales, desarrollo, estabilidad institucional, sistema acusatorio.

\section{SOME NOTES ON THE REASONS FOR THE REFORM OF CRIMINAL PROCEDURE IN LATIN AMERICA}

\begin{abstract}
With the objectives of having a healthier criminal law procedure, today in Latin America, most of the countries modified their criminal codes while others are doing it. In this transition, real international cooperation is needed; support, collaboration, sharing of experience and of best practices etc. That's without putting aside the importance of bilateral and multilateral training delivered by experts from all the fields related to this reform; from human rights to basic citizen involvements passing by the actors of the penal chain. In the development of criminal procedure reform all those countries had successes and all had failures. It seems adequate to start collaborating and have a true discussion to support a better understanding of the implementation of penal systems and building its future evolution. This exchange of knowledge and experiences has to be intelligently and
\end{abstract}


respectfully given and also be received to see what is working elsewhere; adapting them to each particular judicial reality and context, or, be aware of them in order to choose its own ideal solution. On one hand that assistance helps to improve the understanding of the operations of the penal procedure and on the other hand helps to understand the importance of respecting to basic but fundamental legal rights as established in all constitutions of each countries and hence to create institutional stability and confidence for the citizens. Therefore the citizen may see its advantage in security, economic growth both for him and the country and more over to see the advantages of this new penal law system being at the front line of a stronger democracy.

\section{Key words}

Procedural reform, limits and reasons, penal codes, criminal codes, international cooperation, fundamental rights, legal development, institutional stability, accusatorial system

\section{INTRODUCCIÓN}

Destaquemos que en los países latinoamericanos que están en transición hacia el sistema jurídico-penal acusatorio y que adoptan un nuevo derecho, para ellos y en algunos casos similares al que existe en mi país, Canadá, se presenta una inmensa potencialidad de intercambios mutuos. Por una parte, la cuestión del respeto de los derechos de la persona y por otro, la edificación de herramientas básicas con el fin de aplicar en forma adecuada la nueva concepción del sistema y por lo tanto de las normas y garantías que pretende desarrollar. Desde la perspectiva propuesta en el presente documento, se toma el modelo comparativoanalítico de investigación y se examina el caso de México, país que por ejemplo, hizo enormes esfuerzos recientes, lo que incluye modificaciones constitucionales en 2008, incluyendo precisamente, la instauración de un sistema que se orienta hacia un sistema jurídico penal acusatorio. Todo ello sin perjuicio de los procesos que en Latinoamérica ya han sido adelantados y que tienen mayor experiencia en su consolidación, como es el caso de Chile, Colombia, Argentina, Bolivia, Nicaragua, etc.

De todas formas, es claro que el sistema acusatorio plantea problemas prácticos. Aunque sea lógico que una nueva estructura jurídica institucional tenga lugar de manera lenta y gradual, la formación permanece y es un reto importante en todos los niveles tanto en los jueces, los abogados, la sociedad civil como en todos los participantes del sistema jurídico penal. Esto es una combinación de factores que limita un desarrollo rápido de la aplicación de este nuevo sistema.

En México se dice que todo está listo para este cambio y hay mucho interés, tanto de las autoridades como de la sociedad civil para que esta transición tenga lugar cuanto antes. Estos intereses van incluso más allá de las fronteras mexicanas y en este capítulo, el Congreso de los Estados Unidos con la iniciativa de Mérida aprobó más de 1.6 miles millones de dólares para ayudar a dicho país en esta transición.

Sin embargo, debido a varias circunstancias solamente el $10 \%$ de este presupuesto se ha suministrado y se ha gastado hasta la fecha. Una de las razones principales es el reto que coloca el respeto de los derechos de la persona en México y sobre todo, la guerra a los narco traficantes que plantea nuevos fenómenos y retos frente a estos cambios judiciales. Se trata de un ejemplo donde enormes proyectos podrían realizarse. En realidad, la iniciativa de Mérida tiene cuatro piedras angulares. Dos de ellas consisten en la institucionalización de las reformas judiciales y las garantías individuales en el sistema de justicia con miras a su desarrollo y control social para preservar la seguridad y paz ciudadana en general. 
Finalmente, esta mirada a las reformas procesales que se realiza desde la perspectiva de México, plantea como los mecanismos judiciales en el sistema acusatorio deben hacerse en cumplimiento del tamaño de cada país. En este caso, en México se observa que es un gran país y el apoyo que requiere debe hacerse en cumplimiento de sus instituciones. De igual forma, se necesita la cooperación internacional pues la misma debe hacerse solamente, en un contexto de tejido de vínculos sociales, de apoyos sinceros y reales en ámbitos concretos de formación y de apoyo en las mejores prácticas judiciales.

Así mismo, el presente documento recoge la experiencia de Chile a través del Centro de Justicia para las Américas (CEJA), en donde se realizó una evaluación del sistema policial de investigación. De igual forma se recoge la experiencia analizada en visitas realizadas por Canadá a sistemas judiciales de países como Perú, Bolivia, Argentina, República Dominicana y Haití.

Entrando en materia, de manera general, la idea que se tiene de Latinoamérica corresponde más a su pasado. Un pasado glorioso y rico en cultura y una situación política difícil, a veces ligada a la corrupción y a la concentración del poder en las manos de una o varias personas. Desde el principio del siglo XIX, Simón Bolívar se da cuenta de que al conseguir su independencia, todos estos Estados se quedan en una posición vulnerable. Él decía que pequeños tiranos se apropiaban de la madre patria, convirtiéndose arbitrariamente en dictadores, a escala local o nacional ${ }^{1}$.

En calidad de ejemplos, podemos citar al argentino José Mármol, quien durante casi veinticinco años, ejerció un poder absoluto y logró ser el principal dirigente de la Confederación Argentina de 1835 a 1852; al mexicano Porfirio

Ver: CONNIFF, M. Populism in Latin America. University of Alabama Press. Alabama. 1999.
Díaz, de 1876 a 1911; al guatemalteco Estrada Cabrera, de 1898 a 1920; y al cubano Machado, de 1924 a 1933. Todos ellos representaron, ni más ni menos, en esas épocas, la continuación de la explotación por los blancos o los mestizos hacia las poblaciones indígenas. Sin embargo, no es sino hasta después de la Segunda Guerra Mundial que aparecen nuevas dictaduras en Latinoamérica. La proximidad, por no decir injerencia de Estados Unidos hacia Cuba en 1959, trae como consecuencia la Revolución Cubana, ejerciendo también una influencia sobre la evolución política de la región ${ }^{2}$.

Entre las últimas manifestaciones de las más violentas dictaduras tenemos la desaparición de 30,000 personas en Argentina, 4,000 en Chile y cerca de un millar en Brasil. A continuación, siguieron crisis económicas importantes las cuales, a veces de un día para otro, confrontaban a los ciudadanos con la inaccesibilidad a sus propias divisas. Y que decir de los 100.000 muertos en guerras civiles de América central. Recientemente desde los años 40, pasadas las guerras entre traficantes de drogas se crea inestabilidad institucional, particularmente en Colombia con sus 30.000 desaparecidos, "todas son víctimas de grupos armados y las autoridades están buscando sus restos" 3 .

2 Por ejemplo, decía Cordell Hull, Secretario de Estado norteamericano entre los años 1933 y 1944, del dictador de la República Dominicana, Rafael Trujillo: "Quizá sea un hijo de puta, pero es nuestro hijo de puta", quien dominó el país de 1930 hasta su asesinato en 1961, y la lista continúa. Ver: LYNCH, D. J., et al. Caudillos in Spanish America, 1800-1850. Oxford University Press. Oxford. 1992, y THIBAUD, C. Caudillos et pronunciamientos en Colombie. Genèses 61 . París. 2006.

3 Así lo reveló el director de la Unidad de Justicia y Paz de la Fiscalía, Luis González, quien afirmó que desde 1988 hasta 2003 se registró la mayoría de los casos. Diariocrítico - EFE. 20 de Noviembre de 2009. Bogotá. Disponible en: http://www.diariocritico. com/colombia/2009/Octubre/noticias/178685/ colombia-tiene-mas-de-27-mil-desaparecidos.html 
No podemos decir que no es un contexto particular, pues lentamente la llegada de los derechos humanos parece diluir las dictaduras trayendo un bálsamo sobre las cicatrices de América Latina ${ }^{4}$ que vive desde los años ochenta y más activamente desde el año 2000, un profundo cambio democrático; los países de Latinoamérica se incorporan, poco a poco, en una reforma jurídica sin par. Este compromiso no parecía ser el primer punto en la lista de prioridades de la época post dictatorial. A pesar de todo, América Latina ha considerado que la democracia se impondría y tendría que pasar también por una reforma del aparato jurídico ${ }^{5}$.

El presidente Frei, segundo mandatario chileno de la post dictadura, dirigió un importante discurso al Congreso de la República de Chile, en Valparaíso en 1998, respecto a la reforma jurídica:

"Mientras el sistema de administración de justicia en Chile fue diseñado y constituido, en lo fundamental, hacia mediados del siglo XIX, permaneciendo desde entonces sin cambio alguno, la sociedad chilena se ha transformado en un

4 Se utiliza el término América Latina en referencia a los países de Common Law de América del Norte, incluyendo en éstos el territorio de Québec. Punto interesante: se conoce poco sobre el origen del término América Latina. El director del Instituto Francés de Estudios Andinos en Lima, entre 1985 y 1989, Ives Saint-Geours, afirmaba en la edición especial sobre América Latina (322, julio-agosto 2007) de la revista europea l'Histoire que este término apareció en las riberas del Sena en 1856 en la corte de Napoleón III para definir a las naciones "latinas", entonces católicas y que usaban lenguas derivadas del latín, frente a la potencia del mundo protestante y anglosajón, en particular, a los Estados Unidos en pleno surgimiento. Por otra parte, en prácticamente todas las reuniones que organiza España con los países latinoamericanos se usa muy poco este término, ya que prefieren conservar su huella con los términos Iberoamérica ó Hispanoamérica.

5 México, de manera excepcional, apenas empieza con su reforma penal. Uruguay también propuso muy recientemente (julio 2009) un proyecto de reforma penal, así como Panamá. sentido económico y político... El cambio político más importante en Chile ha sido, a su turno, la consolidación del modelo democrático, el que a su vez exige el respeto a los derechos humanos como un principio fundamental de legitimidad... Ambos fenómenos plantean crecientes exigencias al sistema de administración de justicia, que hacen necesaria su modernización, sobre lo cual se ha decantado un importante consenso en el país... Los cambios políticos, a su turno, exigen una justicia accesible, imparcial, igualitaria y que maximice las garantías. La necesidad de prevenir la corrupción supone, a la vez, una activa participación de los ciudadanos en el control del poder y ello aumenta la necesidad de contar con un Poder Judicial eficiente e independiente". 6

Veremos más adelante, que cada uno de los países llevará a cabo esta reforma a su manera. Sin embargo, queda en el espíritu de los países latinos un pasado doloroso, un pasado que deja huellas de sangre, vidas, dolor e injusticia, en la cual, el sistema judicial está ligado a este pasado de corrupción, inequidad y terror, relacionado a veces con el Estado y su incapacidad de apego a las normas jurídicas, lo que incluye las cartas magnas y sus derechos humanos. Esto cuestiona de nuevo, sobre todo entre la población, la credibilidad de los cambios de reforma y suscita también ciertas dudas en relación a su éxito.

Ese pasado doloroso, justifica también para muchos, la urgencia del cambio y el sentimiento de ser forzado por presiones del exterior. No podemos olvidar, el fin de los años setenta y el principio de los ochenta, época en que las cuestiones ligadas a los derechos humanos eran debatidas, negociadas, firmadas y ratificadas. Así mismo, fue una época en la que se adoptaron los principios de democracia -que

6 Ver: Revista de Derecho de Valdivia. V. 10 Agosto 1999. 'Mensaje de SE El Presidente de la República, Don Eduardo Frei Ruiz-Tagle, con que inicia un proyecto de Ley que establece un nuevo código de procedimiento penal'. Boletín $\mathrm{N}^{\circ}$ 1630-07. 
muy a menudo van unidos al acceso a las economías mundiales ${ }^{7}$. Fue claro entonces que América Latina quería liberarse y tenía que democratizarse.

Por tanto, fue decidido atacar lo más inmediato, es decir, el procedimiento criminal que permite juzgar el crimen, corregir la impunidad y ofrecer un clima de seguridad para los ciudadanos; también es un medio de imponer el estado de derecho, controlando de antemano la acción de sus instituciones.

En 1997, Luigi Einaudi ${ }^{8}$, ex secretario adjunto de la OEA e importante diplomático del hemisferio, hablaba del retorno del péndulo histórico de América Latina donde alternaron las democracias nacientes con las dictaduras y que hoy, aunque frágiles todavía, dan el gran paso para instaurar la democracia.

A partir de los años ochenta, la gran mayoría de los países latinoamericanos inician una modificación de su sistema penal, pasa de un sistema inquisitivo a otro más acusatorio, y algunos dejan un sistema mixto?.

$7 \quad$ Promover "el principio de derecho" es un elemento clave en el procedimiento de "la reforma de estado". La reforma legal no tan sólo responde a las demandas del pueblo sino, quizá con mayor importancia para los actores norteamericanos, facilita "la regla mercantil". Como lo dice USAID "los países que tienen sistemas judiciales equitativos proveen ambientes más estables $y$ atractivos para la inversión, ya que ofrecen protección legal para el inversionista. El aumento de la inversión fortalece las economías locales, promueve el crecimiento económico, $y$ crea un ambiente favorable para los inversionistas norteamericanos". También en los años 80, la mayor parte de los países latinoamericanos adoptan una economía neoliberal, dejando a un lado el proyecto de sustitución de importaciones propuesto anteriormente por la CEPAL. Ver: USAID promotes the rule of law in Latin America and Caribbean Democracies. Disponible en: www.usaid.gov/locations/latin_america_caribbean/ democracy/rule/index.html.

8 EINAUDI, Luigi. La politica de seguridad en el hemisferio occidental. Fort Leavenworth. Military Revue. Kansas. 1997.

9 "Promover el principio de derecho ha ido aceptándose ampliamente a través de la región, como un anhelo
Los sistemas inquisitivos variaban considerablemente de un país a otro, y fueron heredados principalmente del pasado, colonial-español y adoptados en el siglo XIX al independizarse, cada país insertando procedimientos adaptados a su propio contexto.

La idea de la reforma se sitúa, en opinión de muchos, en la búsqueda de la eficiencia y de los derechos humanos. Las cárceles estaban llenas, los costos eran muy elevados y los países empezaron a abrir sus puertas al nuevo derecho internacional de los derechos humanos ${ }^{10}$.

necesario para la consolidación de la democracia... el abandono de la reforma del estado y la búsqueda del buen gobierno". En: Domingo, Pilar. "Judicialization of Politics or Politicization of the Judiciary? Recent Trends in Latin America". En: In Democratization. Pub. Routledge. Number 1. Vol. Feb. 2004. p. 105. Varios otros estudios, originados particularmente en Estados Unidos coinciden. Por ejemplo los estudios de: M. Langer, "From Legal Transplants to Legal Translations: the Globalization of Plea Bargaining and the Americanization Thesis in Criminal Procedure" (2004) 45 Harv. Intl. L.J.p.1-27 y M. Unger, "Elusive Reform: Democracy and the Rule of Law in Latin America" (2002) 271, capítulo 2, Boulder pub. London. Carlos Maldonado Curti, Ministro de Justicia en el gobierno Bachelet en Chile y, entonces, responsable de la reforma penal en el país afirmaba: "A partir de la década de los 80 la gran mayoría de los países de América Latina comenzarian a modificar sus sistemas de enjuiciamiento criminal desde un sistema inquisitivo, o inquisitivo mixto, a uno más adversarial", $E n$ : "La Reforma procesal penal chilena: gestación y estado actual del nuevo proceso penal". IX Congreso Internacional del CLAD sobre la reforma del Estado y de la administración Pública. Madrid. 2-5 Nov. 2004. p. 1.

10 Antes de la reforma, la mitad de los individuos en custodia habían sido sentenciados, y la otra mitad estaba esperando sentencia. Eso empezó a cambiar cuando la reforma entró en vigor. De acuerdo a la información más reciente, tres cuartas partes de los presos han sido sentenciados y una cuarta parte espera juicio. Ver: Riego, C. "Abandoning the Inquisitor: Latin America's Criminal Procedure Revolution". 14 Southwestern Journal of Law and Trade in America. (Sw. J. L. \& Trade Am). 2008 p. 339, 352-353. No olvidemos tampoco otras estadísticas: en México sobresalen 90,000 encarcelados por día. 


\section{LAS RAZONES QUE HAN MOTIVADO UNA REFORMA PENAL EN AMÉRICA LATINA}

Los límites del sistema inquisitivo, el retorno a la democracia y la reivindicación ligada a los derechos fundamentales de la persona (entre otros un juicio justo y equitativo en un plazo razonable y la presunción de inocencia), son las razones principales de la reforma del procedimiento penal en América Latina.

\subsection{Las limitaciones del sistema penal en América Latina}

E1 proceso inquisitivo fue introducido por España, tal como sucedió en la gran mayoría de los países de la región, tomando prestados algunos conceptos del sistema alemán, del italiano, y por la fuerza de su historia del sistema francés. A principios del siglo XIX, empezaron a aparecer en Europa continental reformas profundas al procedimiento criminal; en 1808, Napoleón introdujo su Código de Instrucción Criminal que alejó a Francia del modelo inquisitivo representado por la Ordenanza Criminal de 1670, y trasladó algunas ideas del modelo inglés de procedimiento criminal a una jurisdicción civil. Según la tradición inquisitiva de Europa continental, el Código de Instrucción Criminal establecía una investigación, secreta y por escrito previa al juicio, bajo la cual el acusado tenía derechos muy limitados. Sin embargo, inspirado por el modelo inglés, el Código también incluía un juicio oral y público frente a un jurado.

“...Aunque varios actores políticos latinoamericanos promovían la adopción de uno de los modelos mixtos, la mayor parte de los paises de la región finalmente los rechazó. Las élites de América Latina no aceptaron los códigos más liberales, principalmente porque el concepto del jurado no les inspiraba confianza y les disgustaba tanto como los juicios orales y públicos, ya que pensaban que sus pueblos no estaban preparados para ello..."11

Durante el período de la Colonia, este sistema inquisitivo consistía en un procedimiento que daba la facultad de investigar, acusar y condenar, y que estaba concentrado en las manos de una sola persona, es decir el juez, y esto en un procedimiento que tenía como etapa central el expediente escrito y secreto.

En América Latina, el sistema inquisitivo no contribuía a proteger las garantías jurídicas básicas (el "due process"), ni a promover la transparencia la cual, como hoy se sabe, es tan esencial a la administración de la justicia como la justicia misma. Así mismo, el sistema inquisitivo no permitía que todos los actores del proceso penal tuvieran un papel importante, equilibrio tan fundamental para aportar una justicia plena y total. Esos no eran los únicos problemas que conllevaba el sistema inquisitivo de América, sino que eran la principal causa de malestar.

Antes de la naciente reforma constitucional y procesal en México, que recientemente se encuentra en evolución, el proceso penal estaba basado en el sistema inquisitivo que tiene su fuente en el sistema Napoleónico. Es decir, el sistema jurídico penal era completamente escrito; los jueces trabajaban de manera independiente basando su juicio en pruebas llevadas ante ellos de manera escrita por el Ministerio Publico y por la defensa de los acusados. Como todos sabemos y según la historia, este sistema es vulnerable ante intereses poderosos, sobre todo ante organizaciones criminales, contrariamente al sistema acusatorio que por naturaleza es más transparente, permitiendo presentar argumentos orales tanto de la parte del Ministerio Publico como de la parte del abogado defensor,

11 M. LANGER. Revolution in Latin American Criminal Procedure: Diffusion of Legal Ideas from the Periphery. 55 Am. J. Comp. L. 617. 2007. p. 627-628. 
en un juzgado público y ante un juez. Tomando en cuenta que el sistema acusatorio es abierto al público en general, el nuevo sistema jurídico penal puede ser inspeccionado, estudiado, examinado, observado y vigilado por el público en general situación distinta a la tradición enunciada anteriormente. Tomando en cuenta todos estos factores, a continuación se mencionan algunos puntos débiles que fueron objeto esencial de la reforma en Latinoamérica.

\subsection{Concentración de los poderes: el papel del juez}

Uno de los mayores problemas del sistema inquisitivo de América Latina era la concentración del poder en manos de un solo actor, volviéndolo así vulnerable tanto en su persona como en sus decisiones. Es importante comprender que en el modelo inquisitivo el papel del juez era central; se acentúa la diferencia entre justicia penal y justicia civil, privilegiando la posición del juez como representante del interés general, responsable de llevar a cabo la investigación para que triunfe la verdad. Y esto es la gran diferencia entre el sistema inquisitivo y el acusatorio ${ }^{12}$.

Por supuesto, el juez tenía que ser digno de crédito, justo y de buena reputación. Si se desea conocer el origen histórico de este concepto, hay que remontarse hasta la Edad Media, cuando la mayor parte de los países europeos instauraron un sistema inquisitivo de justicia calcado de un modelo eclesiástico, en el cual el

12 "In sum, inquisitorial adjudicators tend to have access in the absence of the parties to a factual written record that is largely unconstrained by rules of admissibility, and adjudicators tend to decide cases primarily on that written record. By contrast, adversarial adjudicators tend to have access only to the evidence the parties give them and that evidence is screened for admission by rules of evidence and then tested for its utility by adversarial challenge, primarily in the form of cross-examination". PACIOCCO, David M. Understanding the Accusatorial System. Canadian Criminal Law Review. August, 2010. p. 7. juez-sacerdote era justo, bueno y sin falla. En el sistema inquisitivo, el juez es un magistrado profesional, dotado de poderes importantes destinados a permitirle dirigir y decidir investigaciones -tanto a cargo como a descargo. En este modelo el juez, más que las partes, tenía la responsabilidad de desarrollar la causa, y también la obligación de "determinar si el acusado era culpable o inocente"13. Difícilmente las partes llegaban a tener un papel en la investigación; la prueba les llegaba después de la acusación y los alegatos se presentaban más tarde y por escrito; este modelo incluye un procedimiento escrito, secreto, no compartido y, por ende, no sujeto a contradicción, y se apoya en la idea de que la justicia represiva no se limita a arbitrar un litigio entre abogados sino que interesa a la sociedad misma: el juez, encargado y responsable de encontrar la verdad, también deja un lugar muy reducido a las partes.

El pasado rígido de América Latina no le permitía al sistema inquisitivo evolucionar dentro del contexto de los derechos humanos ${ }^{14}$. Mientras tanto, Europa continental conoció numerosas reformas en su sistema de procedimiento penal; el sistema, en ciertos momentos, ha sido capaz de adaptarse y lo ha hecho a veces, hasta de la manera radical (Italia, por ejemplo). Hay que mencionar que Europa gozaba de un procedimiento penal completo, lo cual impide hacer

13 PIZZI, William T. "Understanding Prosecutorial Discretion in the United States: The Limits of Comparative Criminal Procedure". In: Ohio State Law Journal. Vol. 54. 1993. 1325-73. p. 1333-1334.

14 "Aunque las naciones latinoamericanas han registrado en décadas recientes una transición hacia la democracia electoral, sus instituciones legales permanecen enturbiadas por legados autoritarios profundamente enraizados". FENSOM, Meredith. Judicial Reform, Military Justice, and the Case of Chile's Carabineros. Tesis presentada en la escuela de graduados de la Universidad de Florida. Master at Arts in the Law and Policy American Program y un documento preparado para entregarse en la Reunión de 2006 de la Asociación de Estudios Latinoamericanos en San Juan, Puerto Rico. Marzo 15-18, 2006. p.7 Disponible en línea: http://www.law. ufl.edu/cgr/lpap/pdf/case_chile_carabineros.pdf 
una comparación entre los dos continentes, aunque en teoría hayan tenido el mismo sistema. Además, si se observa el legado jurídico y judicial dejado a América Latina (y en cierta forma se puede incluir a Haití) ${ }^{15}$ y las divisiones sociales heredadas por todos los países de América Latina, la concentración del poder en las manos de una sola persona, o alrededor de una sola no podía más que contribuir a empantanar el sistema penal, en lugar de beneficiarlo. A causa del papel dominante del juez en el desarrollo del caso y de la importancia de los resultados de su investigación, la fase del juicio en el modelo inquisitivo latino se convirtió en una mera confirmación de los resultados de la investigación y en consecuencia, el principio de moralidad era de poca utilidad ${ }^{16}$. Esta concentración del poder en las manos de un solo actor, agregada al secreto de la investigación y en un contexto político de vulnerabilidad, era

15 La necesidad de una reforma de los códigos es unánime para todos los juristas haitianos. Queda por determinar cuál, y si Haití optará por una reforma que corresponda a la tendencia en el continente americano ó se mantendrá más tradicional, aunque hasta Francia, en el momento en que se escriben estas líneas, a pesar de varias reformas (por ejemplo: su código de instrucción en 1957) propone la eliminación del juez de instrucción, según los términos del reporte del primero de septiembre de 2009 "Rapport du Magistrat Léger”. En 1995, Me Patrick Pierre-Louis, profesor de la Facultad de Derecho de Puerto Príncipe, escribía: "Muchos estudiantes de leyes, numerosos abogados, algunos magistrados y juristas se preguntan respecto a la oportunidad de mantener el título de nuestro Código de instrucción criminal", argumentando, además, que no era suficiente cambiar el nombre de un código de instrucción por uno de procedimiento si la instrucción permanecía y seguía siendo obligatoria. Haití es diferente, siempre lo ha sido, pero entonces no quería proceder a hacer una reforma para volverse como los otros, pero sólo si esta reforma podía llegar a ser una, sino la solución al reforzamiento del estado de derecho haitiano. Ver: CÓDIGO DE INSTRUCCIÓN CRIMINAL, con apuntes de Menan Pierre-Louis. Puerto Príncipe. Presses du D.E.L. 1995

16 DIEHL, James W. "The Introduction of Jury Trials and Adversarial Elements into the Former Soviet Union and Other Adversarial Countries" In: J. Transnat'l L.\& Pol'y:1, 5-28. 2001. p. 26. un campo muy fértil para una falta de responsabilidad, así como para la corrupción.

\subsection{La falta de transparencia y de la responsabilidad imputable}

En el sistema inquisitivo latinoamericano los expedientes eran entonces secretos, es decir, que todo el procedimiento estaba basado en el documento escrito que era poco accesible (a menudo conservado en un resguardo secreto), y sin audiencia; había entonces imposibilidad de contradecirlo. E1 juez investigaba, su asistente acumulaba la prueba y la inscribía en el expediente, el cual conservaba para eventualmente emitir la acusación. Se dijo respecto al poder del juez: "(...) los objetivos represivos de este sistema, que prevalece en la región, conceden canales muy limitados a los acusados y víctimas para controlar los poderes del juez"17. El juez tenía todo bajo su control, delegaba, por no decir relegaba, a un asistente y era difícilmente factible remontar la cadena de eventos en caso de querer contestar. Era prácticamente imposible encontrar al funcionario responsable de tal o cual acción e inclusive, a veces, de encontrar el expediente, ya que era secreto de todos modos. Por lo tanto, era fácil para un funcionario deshacerse de toda responsabilidad. La reforma entonces tenía que atacar al sistema inquisitivo (o mixto, en algunos países), donde la mayor parte del procedimiento estaba ligada al secreto.

"El modelo inquisitivo, como se adaptó a los diferentes sistemas latinos, ha conservado las características europeas fundamentales, pero con cierto número de modificaciones, de origen cultural o politico, en cada sistema nacional de Latinoamérica. $Y$, lo más importante, es que el procedimiento antes

17 PAHL, Michael R. "Wanted: Criminal JusticeColombia's Adoption of a Prosecutorial System of Criminal Procedure". In: 16 Fordham Int'l L.J. 608, 614. 1993. Análisis sobre el sistema inquisitorial de Colombia. 
de las transiciones (...) estaba oculto en la secrecía $y$ casi completamente por escrito"18.

El sistema judicial se volvía la razón de las violaciones a las garantías jurídicas básicas y a los derechos. El sistema inquisitivo de la región aceptaba este hecho, lo repetía y aparecía una inequidad que se volvía costumbre entre los derechos del procurador y los del abogado defensor, durante el juicio. Ellos no se confrontaban con medios iguales; esto resultaba en que el juez, a pesar de su poder, confiaba en subalternos poco calificados, y que no eran juristas ${ }^{19}$.

\subsection{Presencia de la corrupción}

Sorprendentemente, aunque el juez manejara el procedimiento, la rama judicial recibía un mal trato de parte de los políticos ${ }^{20}$. Según varios analistas, no era ni más ni menos que un órgano del ejecutivo con toda su vulnerabilidad, exponiendo la administración de la justicia a una corrupción común y frecuente. El ejecutivo y el legislativo consideraban la tercera rama como su pariente pobre ${ }^{21}$, y se le

18 CAVISE, Leonard L. "When the Inquisitorial and Adversary Systems Collide: Teaching Trial Advocacy to Latin American Lawyers". DePaul College of Law, in Bepress. Berkeley Electronic Press. Legal Series. 2006. Working Paper 1049. 15. Disponible en: http://law. bepress.com/expresso/eps/1049

19 GARCÍA, F.S. "The Nature of Judicial Reform in Latin America and Some Strategic Considerations", 13 Am.U. Int'l. L. Rev.1267. 1998 "En la esfera criminal, los juzgados regionales se han vuelto actores importantes en la violación sistemática de las libertades civiles y de los derechos individuales, a través de la continuidad del sistema inquisitorial criminal".. p. 18. Disponible en: http://lic. law.ufl.edu/ hernandez/ForeignE/garcia.pdf

20 Ver en general: PARIENTE, Alain (Dir.). La séparation des pouvoirs - Théorie contestée et pratique renouvelée. Dalloz. París. 2006.

21 BUSCAGLIA, Edgardo. "Corruption and Judicial Reform in Latin America". In: Policy Studies Journal, 17.4. Instituto Tecnológico Autónomo de México. 1997. Para Perú, AZABACHE, César. Widespread corruption among judicial branch employees... and from the subordination of the legal system to political influence and corruption during the authoritarian rule of Alberto otorgaban pocos recursos financieros. Además, el legislativo y el ejecutivo escogían a los jueces dentro de sus propios partidos políticos, lo que hacía al judicial todavía más vulnerable, tanto económica como políticamente ${ }^{22}$

Fujimori (1990-2002). Extracto del documento en línea: DESHAZO \& VARGAS. Judicial Reform in Latin America. Policy Papers on the Americas, Vol XVII, Estudio 2, Center for Strategic and International Studies (CSIS). Septiembre 2006. Disponible en: csis.org/files/media/csis/pubs/0609_latin_judicial_ reform.pdf. Relacionado, ver también: respecto a Venezuela, PÉREZ PERDOMO, Rogelio. Venezuela's prereform judicial system was marked by inefficiency, widespread corruption, very low level of resources, and the heavy influence of the two leading political parties of the country. Disponible en: http://www.cejamericas.org/ doc/documentos/evaluacionalareformavenezuela rperdomo.pdf y su presentación del 2002 en las conferencias organizadas por el Banco Mundial, disponible en: http://info.worldbank.org/ etools/docs/library/106128/madrid2002/pdf/ rogelioperezperdomo.pdf

22 En Chile se calificaba a la rama judicial como corrupta y tendenciosa. Según una encuesta llevada a cabo en algunos municipios chilenos, la población opinaba que los jueces trataban a los ricos mucho mejor que a los pobres, que los abogados eran corruptos, que los plazos de los procedimientos y las sentencias estaban íntimamente ligados a sumas de dinero, y que no existía más que una sola justicia real, la de los ricos. La conclusión era que el sistema era totalmente corrupto. En el contexto de una conferencia organizada por el grupo SELA de la Universidad de Yale, Jorge Correa Sutil presentó su investigación y apunta:

"Los pobres no tan sólo percibian que la justicia dependía fuertemente de la riqueza, sino que también veían cómo los funcionarios del sistema legal actuaban con discriminación y corrupción que los afectaban. A continuación se mencionan algunas de sus opiniones al respecto:

- $63.5 \%$ dijo que los jueces se comportaban de manera diferente con los ricos que con los pobres.

- 26.3\% dijo que los jueces "no se arreglaban con plata".

- Casi 80\% estuvo de acuerdo en que los abogados eran corruptos y que alargaban las transacciones para sacarle más dinero a la gente.

- Casi las dos terceras partes opinaban que la policía trataba bien a los bien vestidos y mal a los pobres.

- Casi 90\% expresó la opinión de que en Chile habia una justicia para los ricos y otra para los pobres".

Ver a este respecto la encuesta de CORREA SUTIL, Jorge. Access to Justice and Judicial Reforms in Latin 
“(..) el judicial es habitualmente el más comprometido de los tres poderes, lo que lleva a una administración de justicia altamente partidista, donde la manipulación política y la negligencia administrativa y presupuestaria son endémicas"23.

Como el juez tenía todo el poder y delegaba a sus asistentes el cuidado del expediente; éste mismo, a menudo, se quedaba en las manos de un pasante que recibía un magro salario y no tenía a quién rendir cuentas ${ }^{24}$. Era muy común ver desaparecer expedientes ó, en algunos casos, aumentar, modificar o sencillamente extraviar una prueba con el fin de facilitar una liberación de un inculpado, a cambio de dinero; esta falta de transparencia favorecía la corrupción. E1 hecho de que los expedientes no fueran públicos dejaba mucho espacio a la discreción, quizá hasta la arbitrariedad y la manipulación de los documentos escritos.

"El alto nivel de discreción en el manejo de los procesos criminales, la falta de audiencia pública durante el procedimiento para llevar a cabo una discusión simple de la evidencia y las cuestiones legales, aunados al hecho de no someter la decisión final a una tercera parte imparcial comprometen severamente las garantías prevalecientes en las sociedades democráticas modernas. Juzgados regionales han tomado pocas medidas para modificar el statu quo de la justicia criminal, ni en la forma de proponer una revisión completa del sistema criminal o en reforzar las garantias individuales de los acusados. De hecho, la inercia aparente en hacer

America: Any Hope of Equality? Disponible en: http:// islandia.law.yale.edu/sela/jcorre.pdf

23 NUZZI, Laura, et al. "Political Bargaining and Democratic Transitions: A Comparison of Nicaragua and El Salvador". In: 31 J.Latin Am.Stud. 99. 107. 1999.

24 Esta crítica aparece a menudo en los análisis y en todos los países. “... la esencia de la actividad judicial tenía que cambiarse para evitar delegar funciones judiciales a personal sin formación jurídica". Ver: ESPINOZA, C.R. "Abandoning the Inquisitor: Latin America's Criminal Procedure Revolution: Articling: Redesigning Mexico's Criminal Procedure: The States' Turning Point". 15. W. J.L.\& Trade Am. 53. 2008 p. 79. frente a esta situación puede constituir uno de los elementos cruciales que afectan la legitimidad de los juzgados frente a la opinión pública"25.

El fenómeno de la corrupción, junto al papel del juez, se generaliza en el proceso penal donde hay poca gente preparada y a menudo vulnerable por estar mal pagada, dependiendo de dádivas de los políticos y de la elite ${ }^{26}$.

\subsection{El papel secundario del abogado defensor}

Esencialmente, antes de la reforma penal, existían tres actores principales. E1 más modesto de los tres era probablemente el abogado defensor quien venia a intervenir muy tarde en el procedimiento, en cuanto al sistema inquisitivo de América Latina. La tarea principal del procurador de la defensa fue de asegurarse de que el expediente contara con los detalles de la inculpación y de las pruebas; después de la presentación escrita de las pruebas por parte del juez de instrucción, misma que casi siempre era ratificada por el juez del proceso, el derecho de presentar una nueva prueba o a contradecir las existentes en el expediente se podía llevar a cabo solamente si el juez de proceso lo permitía. En el caso de México, un estudio publicado por el Programa de Estudios de Seguridad Pública

$25 \quad$ Véase Supra. No. 17. p. 18.

26 "Los sueldos bajos de los servidores públicos continúan fomentando la corrupción e incompetencia en diversos grados, en todas las instituciones legales. Con frecuencia, los miembros de la policia judicial provienen de áreas muy pobres $y$ tienen poca educación formal; sufren deplorables condiciones laborales y reciben un pago inadecuado por el trabajo peligroso que desempeñan. En lugares como Colombia, los narcotraficantes frecuentemente pueden comprar a la policía con dinero y drogas; entonces, evidencia crucial para juzgar a este tipo de criminales misteriosamente "desaparece". Además, los defensores públicos son, comúnmente, los abogados peor pagados, y el servicio al que pertenecen es la institución que cuenta con menos personal en todo el sistema. Defensores con demasiado trabajo tienden a dar un servicio por debajo de lo esperado a individuos cuyos derechos constitucionales deben resguardar". BISCHOFF, J.L. "Reforming the Criminal Procedure System in Latin America". 9 Tex. Hisp. J.L. \& Pol'y 27. 2003. p. 29. 
y Estado de Derecho, el 3 de Enero del 2010, revela que $82 \%$ de los reclusos no hablaban con el juez en su proceso, en lo que respecta al Distrito Federal y al Estado de México ${ }^{27}$. El abogado defensor no tenía impacto alguno, con la excepción de intervenir al momento de la presentación de la condena. La idea de no poder contradecir las pruebas presentadas desde un principio, estaba orientada hacia los usos y costumbres de la región. Hasta los abogados defensores en los grupos eran reticentes a poner en duda la credibilidad de la investigación del estado, o a estar de acuerdo en que un reto profundo a todas las evidencias proporcionadas por el estado era parte de su deber profesional. Como todos estaban de acuerdo en que la policía haría poco o nada para aportar más elementos a la defensa, los abogados se sentían mejor desarrollando simplemente un caso, independiente de la acusación, y dejar a la fiscalía armar su caso sin interferencia ni participación de la defensa ${ }^{28}$.

\subsection{El Ministerio Público en calidad de burócrata}

La noción de Ministerio Público en América Latina se distingue de la que se encuentra en los países del Common Law. En los países de Common law, el Ministerio Publico funge como defensor de la regla de derecho; en el sistema inquisitivo, el papel del Ministerio Publico es a menudo percibido como el personaje que ratifica la información investigada por los cuerpos policíacos y presentándola

$27 \quad$ Sitio Internet del Programa de Estudios de Seguridad Publica y Estado de Derecho. División de Estudios Jurídicos-CIDE (3 de Enero del 2010). Sin hablar con el juez en su proceso, $82 \%$ de reclusos del DF y del Estado de México, disponible en: http://www. seguridadpublicacide.org. $\mathrm{mx} / \mathrm{cms} /$ pesed-en-losmedios/80-sin-hablar-con-el-juez-en-su-proceso-82de-reclusos-del-df-y-edomex

28 CAVISE, Leonard L. "The Transition from the Inquisitorial to the Accusatorial System of Trial Procedure: Why Some Latin American Lawyers Hesitate". 53 Wayne L. Rev. 785. 2007. p. 806 como prueba, de una manera coherente, ante la autoridad competente. Esta función del Ministerio Publico a menudo se relaciona al papel del Procurador general del Common Law, en los sistemas penales inquisitorios de Latinoamérica, y es percibido como un órgano burocrático del sistema penal. Sin embargo, en regla general, el Ministerio Público es frecuentemente reconocido por las leyes supremas de los países y creado por una ley que le otorga independencia de derecho, de todos los otros actores del sistema judicial. En esta medida, el Ministerio Público fue creado (bajo el nuevo sistema) con autonomía constitucional, para encargarse de tareas de prosecución, así como para resolver casos de imparcialidad y objetividad dentro del área de investigación criminal, y con el fin de reducir la burocracia y profesionalizar la actividad policíaca ${ }^{29}$.

De igual forma, antes de la reforma penal, pocos países tenían un Ministerio Público eficaz. Cuando llegó la modificación a ciertos países, la desaparición del juez de instrucción otorgó mayor poder al Ministerio Público. En la mayoría de los casos, éste último tenía un papel de poca importancia a causa de que el poder de investigación quedaba en manos del juez ${ }^{30}$. Según el profesor chileno Duce, el primer problema que tenía el Ministerio Público, en caso de existir en el sistema de justicia penal, era su ausencia en el procedimiento, es decir, su poder de investigación era vago y poco aplicado. Duce dice que el Ministerio Publico tenía un papel relativo y "reactivo", y que al mismo tiempo, no era más que un burócrata más que aprobaba los

$29 \quad$ BLANCO, R., et al. "Reform to the Criminal Justice System in Chile: Evaluation and Challenges". 2 Loyola University Chicago International Law Review. Loy. U. Chi. Int'1 L. Rev. 2005. p. 253-255.

30 Existe un análisis involucrando a 15 países de América Latina sobre el impacto de la reforma penal en los ministerios públicos. MAIER, Julio, et al. Las Reformas Procesales Penales en América Latina. Publicado por Ad-Hoc, Buenos Aires, en colaboración con la Fundación Konrad Adenauer, 896, 2001. Éste, sin embargo, es del año 2000. 
resultados ${ }^{31}$. En la mejor de las hipótesis el rol de los fiscales se traducía en una colaboración menor a la investigación judicial, por medio de la presentación de algunos antecedentes y la solicitud de diligencias investigativas que el juez de instrucción, a su discreción, podía decretar o negar ${ }^{32}$.

Podemos decir entonces que su función era simbólica sin papel independiente de prosecución, y su poder era utilizado para ratificar las decisiones policíacas para ser rendidas posteriormente al juez. Algunos países suprimieron al juez de investigación desde hace mucho años, como en el caso de México que en nuestros días se propone un nuevo reto, creando una "Fiscalía" cuya función sería la de encabezar al nuevo cuerpo policiaco de investigación. En cuanto al Fiscal, éste servirá de guía jurídico y de representante de la causa ante el juez de la investigación, al igual que la conformación de grupos especializados en temas particulares.

\section{LA REIVINDICACIÓN LIGADA A LOS DERECHOS FUNDAMENTALES}

Antes de la reforma penal en numerosos países de América Latina, existió la conciencia y la necesidad de establecer el respeto de las garantías jurídicas básicas, de elecciones de gobernantes más justas, de gobiernos legítimos, del deseo de prosperar a nivel internacional y de asegurar una economía de libre mercado. El establecimiento del sistema penal acusatorio, el cual esta basado en debates verbales de manera pública, viene con la nueva corriente de

31 DUCE, Mauricio. Sistemas Judiciales, Una Perspectiva Integral Sobre la Administración de Justicia. CEJA. Santiago. 2005. p. 67. Disponible en: http://www. cejamerica.org/doc/documentos/reforma-mp2-final. pdf

32 DUCE, Mauricio. Sistemas Judiciales, "El Ministerio Público en la Reforma Procesal Penal en América Latina". Nov. 2004. p.60. Disponible en: http://www. cejamerica.org/doc/documentos/ceja-duce-reformamp.pdf cambio, con apego al respeto de las garantías individuales.

\subsection{Las garantías jurídicas coercibles pero ejercidas de manera limitada}

Uno de los problemas mayores del sistema inquisitorio, el cual es mencionado en el capítulo anterior, es la falta de respeto a las garantías jurídicas. Por ejemplo, el derecho a ser escuchado, a ser defendido en un plazo razonable, la presunción de su inocencia, la ejecución de órdenes de aprehensión con apego a la constitución, han sido devastados tanto para la mayoría de los ciudadanos, como para el sistema jurídico penal en general, en lo que respecta a América Latina. Hasta hace muy poco no había una tradición de juicios orales, ni oportunidades para el acusado de confrontar a su acusador, refutar un testimonio e impugnar la credibilidad de testigos; por el contrario, el juicio era lento y se llevaba a cabo en secreto. Un funcionario tomaba declaraciones y preparaba el testimonio por escrito para que el juez de la investigación lo leyera después; el acusado era un objeto de una investigación, más que un sujeto con derechos humanos y civiles. No era sorprendente que el sistema inquisitivo fuese permisivo también, eventualmente, frente a la tortura y otras violaciones a los derechos humanos. Dicho sistema no facilitaba las vías de derecho, la transparencia ni la participación ${ }^{33}$.

La realidad es que solamente las personas con más recursos económicos contrataban con la oportunidad de contratar un abogado que trabajase de la mano con funcionarios de la justicia acelerando y facilitando la liberación del inculpado de manera pronta y expedita. Por el contrario, donde no había recursos, tampoco había garantías constitucionales respetadas. Las circunstancias para las personas

33 COOPER, J.M. "Proyecto Acceso: Using Popular Culture to Build the Rule of Law in Latin America". In: Rutgers Journal of Law \& Public Polic. 2008. p. 2. 
sin recursos, eran aun peor, puesto que los presuntos inculpados pasaban días, semanas, y a veces hasta años, sin juicio alguno. Los más vulnerables, que constituían la mayoría de los ciudadanos, poblaban las cárceles por crímenes no tan graves.

"Aunque parezca increible, setenta por ciento de los reclusos de las prisiones latinoamericanas está esperando juicio. Realmente, la duración del encarcelamiento previo al juicio, a menudo excede la sentencia máxima a que se haría acreedor el acusado en caso de ser convicto del crimen por el que todavía no ha sido procesado" ${ }^{34}$ y "Arrestos ilegales, golpizas y tortura son también problemas comunes para los niños pobres que viven en las calles de países como Brasil. De hecho, ochenta por ciento de la población de las cárceles de Sao Paulo fue calificado, alguna vez, como niño de la calle"35.

Como los datos demuestran, en lo que respecta el sistema inquisitorio, la investigación es lenta y el juicio se lleva a cabo mucho tiempo después de la misma. En general y de acuerdo a la historia del sistema de justicia penal en América Latina, el procedimiento se oponía al respeto de las garantías jurídicas de las personas. La víctima no tenía, prácticamente participación alguna, ni derecho durante el juicio. En los pocos casos en donde todavía sobrevive, el sistema inquisitivo en América Latina, se denomina "sistema escrito", no tan sólo a raíz de la gran cantidad de alegatos y reportes escritos, sino también a causa de que tradicionalmente se ha puesto un gran énfasis en la formalidad en todas las etapas del juicio; todos los documentos mencionados son objeto de archivo y "cosidos unos a otros". El juez revisa este

34 BISCHOFF, James L. "Reforming the Criminal Procedure System in Latin America". 9 Tex. Hisp.J.L. \& Pol'y 27. 2003. p. 40. SEITLES, M.D. "Effect of the Convention on the Rights of the Child upon Street Children in Latin America: a Study of Brazil, Columbia and Guatemala". 16. Buff. Jour. Pub. Int. Law 159. 1997. p. 186.

35 SEITLES, M.D. Op. Cit. p. 186. expediente voluminoso, para entonces llegar a sus conclusiones basándose en la información escrita $^{36}$.

El juez, en estos casos, emitía su sentencia antes de que el expediente se entregara al juez del proceso o al mismo demandante. Sin objeción alguna del juez de la investigación, las partes no eran escuchadas y en general, todo terminaba en detención preventiva sin haberse presentado defensa alguna para el presunto delincuente; el acusado, sin acceso a contra-interrogatorio alguno, soportaba todo el peso de las pruebas. Dicho de otra manera, el acusado podía seguir encerrado hasta el momento de su juicio.

Podemos decir que uno de los problemas fundamentales del procedimiento inquisitivo en algunos países de América latina, es que el juez del proceso no hace más que ratificar la decisión del juez. El defecto se encuentra precisamente en que la investigación es secreta y por consiguiente, la toma de decisiones se realiza, sin que haya habido representación jurídica para los acusados, lo que conlleva a decisiones jurídicas sobre la libertad de los individuos sin poder presentar una defensa plena y apropiada. Ciertos países, han ido aun mas lejos, presentando acusaciones finales hechas por el juez de la investigación sin que un juez de proceso se haya presentado jamás ${ }^{37}$. Tanto el juez de la investigación como el del proceso, colegas en la misma corte (jurisdicción-juzgado), no rendían cuentas a ningún procurador independiente de ellos. Por otro lado, el abogado de la defensa no conocía ni las pruebas, ni la investigación. É1 intervenía por escrito sin posibilidad de realizar contra-interrogatorio alguno puesto que en términos prácticos, no había ninguna utilidad en

36 Ver FIELD, Martha A, et al. "Legal Reform in Central America: Dispute Resolution and Property Systems". Harvard University Press. 2001. p. 22-23.

37 Special Report: Presumed Guilty? Criminal Justice and Human Rights in Mexico. 24, F ordham Int'1. L.J. 801, 831. 2001. 
contestar una decisión final. "Los procedimientos se conocen como "no adversariales" porque cada uno de los participantes en esta fase es independiente, ya sea que hayan sido designados como "rapporteurs" o jueces" 38 .

Como se puede ver, a priori, en la reforma penal de Latinoamérica, hay que estudiar con detenimiento el papel de los actores, en particular de los jueces, crear un procurador público e introducir la "audiencia pública". No hay que olvidar que el concepto de la falta de aplicación de las garantías individuales, lo cual es una réplica de la inquisición es opuesto a la defensa del acusado que es celosamente defendida en los países del Common Law.

\subsection{La reivindicación ligada a los derechos fundamentales y la participación de la sociedad}

Es la sociedad civil quien debe reivindicar el respeto a las reformas jurídicas, en particular, en el ámbito de la administración de la justicia y en el respeto de los derechos fundamentales de un país. Uno de los grandes intelectuales de la reforma penal de América Latina, el profesor Alberto Binder de la Universidad de Buenos Aires, siempre afirmó que la sociedad civil en Latinoamérica tiene la viva capacidad de buscar mejores coyunturas en derecho, a pesar del adelgazamiento que ha sufrido. En una entrevista, el profesor Binder dijo:

"Creo que las organizaciones de la sociedad civil cumplen hoy varios papeles importantes. Uno de ellos ha nacido en América Latina durante la última década, impulsado por el importante trabajo de un conjunto de organizaciones que, con un lenguaje común, tienen la capacidad de incidir en las politicas judiciales. Esto ha sido muy positivo. $Y$ ésta es la primera función: mantener y lograr que nuestros estados tengan politicas judiciales permanentes y no que cada cincuenta años tengamos

$38 \quad$ Leonard L. Cavise. Véase Supra No.. 17. p. 5. que hacer esta tarea ciclópea de cambiar todo el sistema" ${ }^{\prime \prime 3}$.

En un contexto donde se busca la evolución del sistema judicial y del Estado de derecho, el respeto de las garantías jurídicas en los juicios, son el corazón del proceso de reforma. Esto ocurrió en Argentina, cuando ya no se quería volver a vivir juicios sin fin, secretos o parodias de juicios. Ya no se quería saber de desaparecidos, sino de gente condenada o liberada que recibiese sentencias y no favores; la sociedad civil demandaba justicia.

La reforma judicial ha sido considerada por largo tiempo como un prerrequisito para la consolidación de la democracia y para un desarrollo sustentable en América Latina. En la mayor parte de los países en la región, empezó en la última década del siglo XX con instituciones judiciales débiles, políticamente vulnerables e ineficientes. Pocos fueron capaces de mantener el poder ejecutivo estable, de garantizar la observancia efectiva de los derechos humanos y civiles básicos, de crear un ambiente favorable para el desarrollo económico, especialmente para inversión extranjera y nacional o de proveer la seguridad básica a los ciudadanos. Estaban sufriendo por códigos criminales anticuados, mal organizados, y dependiendo de tribunales endebles, de la preparación y retribución inadecuada de los jueces, funcionarios judiciales policías, de procedimientos legales que minimizaban la transparencia y casi siempre, de condiciones carcelarias deplorables ${ }^{40}$.

De igual forma, además de algunos tecnócratas, son algunos intelectuales-académicos animados y apoyados por las presiones del exterior, quienes han venido a proponer a las

39 Entrevista con el profesor argentino BINDER, Alberto. La reforma judicial: Para evitar la sociedad violenta y la democracia autoritaria. Disponible en: http://www.justiciaviva.org.pe/entrevis/03.pdf

40 DESHAZO, Peter, et al. "Judicial Reform in Latin America: An Assessment”. En: Supra No. 20. p. 6. 
nuevas democracias, un proyecto de democratización jurídica y judicial. Carlos Maldonado, al comentar la experiencia chilena, afirmaba que la reforma es un proceso que ha empezado en la sociedad civil con la importante contribución de los intelectuales del exterior del país, posteriormente ratificada por el ejecutivo del país, quien vio la reforma como una buena plataforma política ${ }^{41}$.

Sin embargo y pese a todas estas circunstancias, la reforma del procedimiento penal se ha generalizado en América Latina. Importantes reformas han tenido lugar, por ejemplo en Córdoba, Argentina (1991), en Guatemala (1994), en Costa Rica (1998) ${ }^{42}$, en la provincia de Buenos Aires, Argentina (1999), en el Salvador, en Paraguay y Venezuela (2000), en Chile -(de manera gradual, ${ }^{43}$ empezando en 2001),

41 En: Supra No. 9. Maldonado, p. 3 "Principalmente mediante un trabajo de académicos de algunas universidades nacionales en conjunto con algunas ONGs que desde el comienzo han trabajado en la reforma procesal penal".

42 Costa Rica, en 1973, había adoptado un Código del procedimiento penal, basado en el Código de la Provincia argentina de Córdoba de 1939, cuya referencia era el viejo código italiano bajo el nombre de Rocco, proveniente del contexto autoritario. En pocas palabras, cada cual tenía su propio modelo rígido. Paraguay, por su lado, tenía un código heredado de la Alemania Nazi. El Código costarricense de 1973 establecía un sistema "mixto", en donde la primera parte del juicio tenía rasgos claramente inquisitivos (proceso escrito, juez de instrucción, secreto de la investigación, sospechoso como simple objeto del proceso, etc.), mientras que la fase del juicio se quedaba esencialmente acusatoria (audiencia pública, contestación de las partes, acusación formal, etc.). Pero las debilidades del sistema saltaban a la vista: juicios largos, una cantidad apabullante de material escrito reproducido durante la etapa del juicio oral, el alejamiento de las partes del proceso respecto de la administración de justicia.

43 Primera etapa: Regiones de Coquimbo y Araucania, 16 de diciembre del 2000.

Segunda etapa: Regiones de Antofagasta, Atacama y Maule, 16 de octubre del 2001.

Tercera etapa: Regiones de Taracapa, Aysen y Magallaes, 16 de diciembre del 2002.

Cuarta etapa: Regiones de Valparaíso, O'Higgins, BioBio y Los Lagos, 16 de diciembre del 2003. en Bolivia y Ecuador (2002), en Honduras y Nicaragua (2005), en Colombia -de manera gradual (2003-2004 -2005-2008), en la República Dominicana (2006), en Perú - (también en forma gradual), en la Provincia de Chubut en Argentina (2007), en el Estado de Chihuahua, en México, en el Distrito Federal de México, en el Estado de Oaxaca (2008), y actualmente empieza un debate sobre la reforma en el Estado federal de Argentina, y en Panamá ${ }^{44}$.

Algunos países empezaron a integrar los grandes principios de la reforma en su constitución. Seguido a ello, se observó un gran movimiento legislativo imponiendo la adopción de códigos penales y de procedimiento penal en consecuencia de la reforma constitucional. Es decir, se instauró lo que en América Latina se denominó como la "oralidad", refiriéndose a la posibilidad para las partes de defenderse, de tener acceso a las pruebas, debatirlas y de presentar pruebas en nombre de la justicia, de la transparencia jurídica y sobre todo, de respeto a sus respectivas constituciones y garantías jurídicas, todo ello en forma oral lo que agiliza los procedimientos. Desde entonces, se ha creado un nuevo ministerio público, otorgándole un poder de investigación real, redefiniendo el papel del juez de conocimiento y de control de garantías; se ha recreado la figura de nuevos defensores públicos, se le otorgado un lugar a la víctima y a los testigos así como su protección mediante sistemas y programas específicos y se han creado nuevos códigos penales y de procedimientos penales en casi cada lugar de Latinoamérica.

Con el nuevo sistema de justicia penal en la mayoría de los países latinoamericanos, el juez se ve obligado a tomar su distancia de las investigaciones criminales, asumiendo una actitud mucho más pasiva. Es entonces que el juez se

\footnotetext{
Quinta y última etapa: región metropolitana (Santiago), 16 de junio del 2005.

44 Ver al respecto el texto de Leonard L. Cavise. Ob. Cit.
} 
vuelve un observador, teniendo como tareas más importantes, las de proteger los derechos de las partes -principalmente los del acusado-, tomando decisiones sobre cuestiones esenciales del proceso, lo que incluye la imposición de medidas preventivas, definiendo la situación jurídica del acusado. "Así, el papel de los jueces se transforma en un papel de protección, de guardián y protector de nuestras débiles democracias" 45 .

A partir de este momento, el juez viene perdiendo la posibilidad de controlar oficiosamente el desarrollo del proceso, lo cual no es ni será una tarea fácil. Alguien dijo al respecto que:

"(Los jueces) estaban acostumbrados a hacerse cargo de la mayor parte de los elementos del proceso: supervisar la investigación, llamar a cualquier testigo que quisieran, en el orden que quisieran, y después hacer todas o la mayor parte de las preguntas. Para un juez el ser "relegado" al papel de árbitro, apenas dando opiniones sobre objeciones o puntos legales, siendo visto, más no oído, hasta que se presentara un problema, representaba quizá un choque demasiado dificil de absorber en las pocas semanas de los programas. Una y otra vez, las simulaciones eran desviadas por un "juez" (tuviera o no el nombramiento), quien quería encontrar de inmediato lo que él, o ella, quería saber y no estaba dispuesto(a) a dejar que la instrucción tomara su curso. En esta situación, el juez insistiría en cuestionar, argumentando con los abogados, introduciendo elementos de evidencia que ninguna parte habia sometido, o simplemente comentaría muy prematuramente sobre la credibilidad"46.

A pesar de todo, varios estudios concluyen que la cultura está todavía muy lejos del cambio para que la reforma penal sea considerada como un éxito. Así lo detalla el primer Director del Centro de estudios de la Justicia de

45 LOAYZA, María de los Ángeles. Condiciones de la reforma del procedimiento legal: de la teoría a la práctica: la experiencia boliviana. Seminario sobre el Estado de derecho y reforma penal. Ëcole Nationale de la Magistratura. Port-au-Prince. Haití.

$46 \quad$ Ver: Supra No. 26. p. 807. las Américas, Juan Enrique Vargas Vianco ${ }^{47}$, tomando en cuenta el ejemplo de Bolivia y de Honduras para quien, según el estudio del Banco Mundial ${ }^{48}$, en el capítulo sobre la administración de la justicia, se identifican algunos problemas de la reforma como los siguientes: la falta de formación en el soporte técnico; en el manejo de los recursos humanos que incluye la falta de estabilidad de los mismos; la falta de una infraestructura inadecuada, así como una inadecuada distribución de las causas. Este estudio menciona también que los actores siguen procediendo con una perspectiva inquisitiva, a pesar de estar en un marco jurídico acusatorio. El reporte anterior, hace también referencia a que la formación debe incluir, a partir de ahora, un cambio de mentalidad hacia la cultura del sistema acusatorio, permitiendo a los actores y a la población aceptar, que aunque el nuevo sistema parezca menos severo, la aplicación de la justicia mejora en un sentido realista. La Comisión se da cuenta de la dificultad de aceptar el cambio, en razón de que el antiguo sistema les inspira confianza. Es difícil entender que el hecho de no encarcelar preventivamente a un posible criminal, no implique necesariamente una injusticia y que permitir a las partes debatir con apego a la norma jurídica, no resulta automáticamente en una pérdida de tiempo o en

$47 \quad$ En muchas ocasiones, el Ceja está haciendo estadísticas sobre algunos países, mostrando notablemente que aunque se presentan muchos desafíos se puede con la reforma penal disminuir muchas situaciones en relación con la justicia. Ver: VARGAS, Juan Enrique. La Nueva Generación de Reformas Procesarles Penales en Latinoamérica. Ponencia Presentada al Congreso Internacional de Derecho Penal. - VII Jornada sobre Justicia Penal. UNAM. México. Junio 2006. Disponible en: www.juridicas.unam. $\mathrm{mx} /$ sisjur/ penal/pdf/11-509s.pdf

48 COMISIÓN INTERAMERICANA DE DERECHOS HUMANOS (IACHR) Y EL BANCO MUNDIAL. LAS REFORMAS A LA ADMINISTRACIÓN DE JUSTICIA EN HONDURAS Y BOLIVIA. Razones que han obstaculizado su éxito y como enfrentarlas. Octubre 2008. 198 páginas. Disponible en: http://www.dplf.org/uploads/1227112057.pdf 
una debilidad al juzgar ${ }^{49}$. Parece que la forma en la cual las instituciones administran los casos sigue la misma lógica: cada participante actúa individualmente, aislado del resto de la institución, asume la responsabilidad por un cierto número de casos, desatendiendo cuestiones que no se refieren a los suyos ${ }^{50}$.

Esa perspectiva es bastante cuestionable, tomando en cuenta que por un lado el sistema inquisitivo tuvo siempre garantías individuales raramente hechas valer, con la excepción de las pocas minorías que podían costearse abogados sagaces, técnicamente preparados y con poder de influencia ante los actores del sistema judicial para mover los intereses de sus clientes de manera rápida y efectiva. Por otro lado, el nuevo sistema jurídico penal, orientado hacia el sistema acusatorio en oposición del sistema inquisitivo, tiene como uno de los objetivos fundamentales de rendir justicia de manera pronta y expedita para todos los acusados. Por ende, el sistema acusatorio que tiene su raíz en el Common law, requiere una perspectiva diferente. De manera obvia, la formación permanente para todos los actores del sistema jurídico penal, es igualmente fundamental y por lo tanto merece de un tratamiento aparte

49 IACHR. Access to Justice and Social Inclusion: "The road towards strengthening democracy in Bolivia". Washington D.C. 2007. Disponible en: http://www. cidh.org/pdf\%20files/BOLIVIA.07.ENG.pdf. Por ejemplo, el reporte menciona en su párrafo 18: "la Comisión encontró que una de las principales debilidades del Ministerio Público es la disparidad y la falta de coordinación que existen en los criterios para el procedimiento criminal asi como la falta de controles efectivos sobre la actuación de los procuradores; especialmente cuando se trata de rechazar quejas, de dar prioridad en la investigación de casos, de aplicar soluciones alternativas o el alcance de la discreción, de escoger casos para juicio oral e, inclusive, los parámetros para designar a los procuradores según el tipo de crimen".

50 MANNING, Nick, et al. Las reformas a la administración de Justicia en Honduras y Bolivia, razones que han obstaculizado su éxito y como enfrentarlas, fundación para el debido proceso Legal. Banco Mundial. Oct. 2008. Anexo 2. p.175. Disponible en: http://www.dplf.org/ uploads/1227112057.pdf y en extenso en un próximo artículo en donde se pueda avanzar en la participación activa de la población en el nuevo sistema de justicia penal; la formación a nivel intersectorial para funcionarios jueces, defensores, universitarios y juristas en general; los elementos que debe considerar el Ministerio Público para lograr autonomía e independencia; la implementación y ampliación de los mecanismos alternativos de solución de conflictos; los programas de protección a víctimas y testigos entre otros aspectos relevantes del sistema.

\section{CONCLUSIÓN}

Desde el punto de vista político el gran escenario de la reforma penal puede tener como marco de fondo a muchos países. En tal sentido, el hemisferio es una vasta zona geográfica y conoce una transformación social y política sin precedentes con un potencial económico incalculable. Como le dijo el presidente Calderón de México con ocasión del bicentenario de México, "la reforma penal no va a arreglar el problema de la violencia", no va a hacerlo sólo, pero es un herramienta mi importante que tienen a dentro las posibilidades de anunciar la paz ciudadana, el respecto del derecho de cada humanos en su país y a fuera y el respecto del estado de derecho con todo sus beneficios. Desde el final de los regímenes dictatoriales, los pueblos aspiran a la democratización de sus países, a la estabilidad de sus instituciones y a la lucha contra la corrupción y la impunidad. Este nuevo impulso incluye la reforma de su sistema penal. Ahora somos un solo, un gran hemisferio que necesita ayudarse y respectarse para hacer una America de derecho y de paz por cada ciudadano. Es la hora de la implementación.

América Latina está en una etapa de transformación, los sistemas evolucionan y se acercan a la idea de un papel global que inspire mayor seguridad y respeto a sus realidades. Estos países adoptan la reforma como una de sus 
prioridades hacia la democratización, el buen gobierno y la edificación del estado de derecho.

En principio, la reforma es un éxito ya que permite a los Estados dotarse de herramientas, cuando menos en derecho penal, más transparentes y deja vislumbrarse en el horizonte, y para los años venideros, una confianza en la administración de la justicia de parte de la población. Los derechos fundamentales que hemos aprendido a conocer, vienen a confirmar para estos países la importancia de que la apariencia de justicia es una noción que jamás se vuelve costumbre y que siempre hay que reforzar. Pero hay que invertir. La ayuda internacional es primordial; una ayuda original y real que respete de la grandeza de los países hermanos del mismo hemisferio.

\section{BIBLIOGRAFÍA}

AZABACHE, César. Widespread corruption among judicial branch employees... and from the subordination of the legal system to political influence and corruption during the authoritarian rule of Alberto Fujimori (1990-2002). Extracto del documento en línea: DESHAZO \& VARGAS. Judicial Reform in Latin America. Policy Papers on the Americas, Vol XVII, Estudio 2, Center for Strategic and International Studies (CSIS). Septiembre 2006. Disponible en: csis.org/files/ media/csis/pubs/0609_latin_judicial_reform. pdf.

BINDER, Alberto. La reforma judicial: Para evitar la sociedad violenta y la democracia autoritaria. Disponible en: http://www.justiciaviva. org.pe/entrevis/03.pdf

BISCHOFF, J.L. "Reforming the Criminal Procedure System in Latin America". 9 Tex. Hisp. J.L. \& Pol'y 27. 2003.

BLANCO, R., et al. "Reform to the Criminal Justice System in Chile: Evaluation and Challenges". 2 Loyola University Chicago International Law Review. Loy. U. Chi. Int'1 L. Rev. 2005.
BUSCAGLIA, Edgardo. "Corruption and Judicial Reform in Latin America". In: Policy Studies Journal, 17.4. Instituto Tecnológico Autónomo de México. 1997.

"The Transition from the Inquisitorial to the Accusatorial System of Trial Procedure: Why Some Latin American Lawyers Hesitate". 53 Wayne L. Rev. 785. 2007.

CAVISE, Leonard L. "When the Inquisitorial and Adversary Systems Collide: Teaching Trial Advocacy to Latin American Lawyers". DePaul College of Law,in Bepress. Berkeley Electronic Press. Legal Series. 2006. Working Paper 1049. 15. Disponible en: http://law.bepress.com/ expresso/eps/1049

CÓDIGO DE INSTRUCCIÓN CRIMINAL, con apuntes de Menan Pierre-Louis. Puerto Príncipe. Presses du D.E.L. 1995

COMISIÓN INTERAMERICANA DE DERECHOS HUMANOS (IACHR) Y EL BANCO MUNDIAL. LAS REFORMAS A LA ADMINISTRACIÓN DE JUSTICIA EN HONDURAS Y BOLIVIA. Razones que han obstaculizado su éxito y como enfrentarlas. Octubre 2008. 198 páginas. Disponible en: http://www. dplf.org/uploads/1227112057.pdf

CONNIFF, M. Populism in Latin America. University of Alabama Press. Alabama. 1999.

COOPER, J.M. "Proyecto Acceso: Using Popular Culture to Build the Rule of Law in Latin America". In: Rutgers Journal of Law \& Public Polic. 2008.

CORREA SUTIL, Jorge. Access to Justice and Judicial Reforms in Latin America: Any Hope of Equality? Disponible en: http://islandia.law. yale.edu/sela/jcorre.pdf

DESHAZO, Peter, et al. "Judicial Reform in Latin America: An Assessment". En: Supra No. 20.

Diariocrítico - EFE. 20 de Noviembre de 2009. Bogotá. Disponible en: http:// 
www.diariocritico.com/colombia/2009/ Octubre/noticias/178685/colombia-tienemas-de-27-mil-desaparecidos.html

DIEHL, James W. "The Introduction of Jury Trials and Adversarial Elements into the Former Soviet Union and Other Adversarial Countries" In: J. Transnat'l L.\& Pol'y:1, 5-28. 2001.

DOMINGO, Pilar. "Judicialization of Politics or Politicization of the Judiciary? Recent Trends in Latin America". En: In Democratization. Pub. Routledge. Number 1. Vol. Feb. 2004.

DUCE, Mauricio. Sistemas Judiciales, "El Ministerio Público en la Reforma Procesal Penal en América Latina". Nov. 2004. Disponible en: http://www.cejamerica.org/doc/documentos/ceja-duce-reforma-mp.pdf

Sistemas Judiciales, Una Perspectiva Integral Sobre la Administración de Justicia. CEJA. Santiago. 2005. Disponible en: http://www. cejamerica.org/doc/documentos/reformamp2-final.pdf

EINAUDI, Luigi. La politica de seguridad en el hemisferio occidental. Fort Leavenworth. Military Revue. Kansas. 1997.

ESPINOZA, C.R. "Abandoning the Inquisitor: Latin America's Criminal Procedure Revolution: Articling: Redesigning Mexico's Criminal Procedure: The States' Turning Point". 15.W. J.L.\& Trade Am. 53. 2008.

FENSOM, Meredith. Judicial Reform, Military Justice, and the Case of Chile's Carabineros. Tesis presentada en la escuela de graduados de la Universidad de Florida. Master at Arts in the Law and Policy American Program. Disponible en línea: http://www.law.ufl.edu/cgr/ lpap/pdf/case_chile_carabineros.pdf

FIELD, Martha A, et al. "Legal Reform in Central America: Dispute Resolution and Property Systems". Harvard University Press. 2001
GARCÍA, F.S. "The Nature of Judicial Reform in Latin America and Some Strategic Considerations", 13 Am.U. Int'l. L. Rev.1267. 1998 "En la esfera criminal, los juzgados regionales se han vuelto actores importantes en la violación sistemática de las libertades civiles y de los derechos individuales, a través de la continuidad del sistema inquisitorial criminal". Disponible en: http://lic.law.ufl. edu/ hernandez/ForeignE/garcia.pdf

IACHR. Access to Justice and Social Inclusion: "The road towards strengthening democracy in Bolivia". Washington D.C. 2007. Disponible en: http://www.cidh.org/pdf\%20files/BOLIVIA.07.ENG.pdf.

LANGER, M. "From Legal Transplants to Legal Translations: the Globalization of Plea Bargaining and the Americanization Thesis in Criminal Procedure". 45 Harv. Intl. L.J.p.1-27. 2004.

Revolution in Latin American Criminal Procedure: Diffusion of Legal Ideas from the Periphery. 55 Am. J. Comp. L. 617. 2007.

LYNCH, D. J., et al. Caudillos in Spanish America, 1800-1850. Oxford University Press. Oxford. 1992.

LOAYZA, María de los Ángeles. Condiciones de la reforma del procedimiento legal: de la teoría a la práctica: la experiencia boliviana. Seminario sobre el Estado de derecho y reforma penal. Ëcole Nationale de la Magistratura. Port-au-Prince. Haití.

MAIER, Julio, et al. Las Reformas Procesales Penales en América Latina. Publicado por Ad-Hoc, Buenos Aires, en colaboración con la Fundación Konrad Adenauer, 896, 2001. Éste, sin embargo, es del año 2000.

MALDONADO CURTI, Carlos. "La Reforma procesal penal chilena: gestación y estado actual del nuevo proceso penal". IX Congreso Internacional del CLAD sobre la reforma del Estado $y$ de la administración Pública. Madrid. 2-5 Nov. 2004. 
MANNING, Nick, et al. Las reformas a la administración de Justicia en Honduras y Bolivia, razones que han obstaculizado su éxito y como enfrentarlas, fundación para el debido proceso Legal. Banco Mundial. Oct. 2008. Anexo 2. Disponible en: http://www.dplf.org/uploads/1227112057. pdf

NUZZI, Laura, et al. "Political Bargaining and Democratic Transitions: A Comparison of Nicaragua and E1 Salvador". In: 31 J.Latin Am.Stud. 99. 107. 1999.

PACIOCCO, David M. Understanding the Accusatorial System. Canadian Criminal Law Review. August, 2010.

PAHL, Michael R. "Wanted: Criminal JusticeColombia's Adoption of a Prosecutorial System of Criminal Procedure". In: 16 Fordham Int'l L.J. 608, 614. 1993.

PARIENTE, Alain (Dir.). La séparation des pouvoirs - Théorie contestée et pratique renouvelée. Dalloz. París. 2006.

PÉREZ PERDOMO, Rogelio. Venezuela's prereform judicial system was marked by inefficiency, widespread corruption, very low level of resources, and the heavy influence of the two leading political parties of the country. Disponible en: http:// www.cejamericas.org/doc/documentos/ evaluacionalareformavenezuela_rperdomo.pdf y su presentación del 2002 en las conferencias organizadas por el Banco Mundial, disponible en: http://info.worldbank.org/etools/docs/ library/106128/madrid2002/pdf/rogelioperezperdomo.pdf

PIZZI, William T. "Understanding Prosecutorial Discretion in the United States: The Limits of Comparative Criminal Procedure". In: Ohio State Law Journal. Vol. 54. 1993. 1325-73

Programa de Estudios de Seguridad Pública y Estado de Derecho. División de Estudios Jurídicos-CIDE (3 de Enero del 2010). Sin hablar con el juez en su proceso, $82 \%$ de reclusos del DF y del Estado de México, disponible en: http:// www.seguridadpublicacide.org. $\mathrm{mx} / \mathrm{cms} /$ pesed-en-los-medios/80-sin-hablar-con-el-juezen-su-proceso-82-de-reclusos-del-df-y-edomex

Revista de Derecho de Valdivia. V. 10 Agosto 1999. 'Mensaje de SE El Presidente de la República, Don Eduardo Frei Ruiz-Tagle, con que inicia un proyecto de Ley que establece un nuevo código de procedimiento penal. Boletín $\mathrm{N}^{\circ} 1630-07$.

RIEGO, C. "Abandoning the Inquisitor: Latin America's Criminal Procedure Revolution". 14 Southwestern Journal of Law and Trade in America. (Sw. J. L. \& Trade Am). 2008.

SEITLES, M.D. "Effect of the Convention on the Rights of the Child upon Street Children in Latin America: a Study of Brazil, Columbia and Guatemala". 16. Buff. Jour. Pub. Int. Law 159. 1997.

Special Report: Presumed Guilty? Criminal Justice and Human Rights in Mexico. 24, F ordham Int'1. L.J. 801, 831. 2001.

Supra No. 9.

Supra No. 17.

Supra No. 26.

THIBAUD, C. Caudillos et pronunciamientos en Colombie. Genèses 61. París. 2006.

UNGER, M. "Elusive Reform: Democracy and the Rule of Law in Latin America". 271, capítulo 2, Boulder pub. London. 2002.

USAID promotes the rule of law in Latin America and Caribbean Democracies. Disponible en: www. usaid.gov/locations/latin_america_caribbean/ democracy/rule/index.html.

VARGAS, Juan Enrique. La Nueva Generación de Reformas Procesarles Penales en Latinoamérica. Ponencia Presentada al Congreso Internacional de Derecho Penal. - VII Jornada sobre Justicia Penal. UNAM. México. Junio 2006. Disponible en: www.juridicas.unam.mx/ sisjur/penal/pdf/11-509s.pdf 